\title{
MEGARA Spectrograph Optics
}

\author{
Carrasco, E. ${ }^{1}$, Sánchez-Blanco, E. ${ }^{2}$, García-Vargas, M.L. ${ }^{2}$, Gil de Paz, A. ${ }^{3}$, Páez, G. ${ }^{4}$, Gallego, J. ${ }^{3}$, \\ Sánchez, F.M. ${ }^{5}$, Vílchez, J.M. ${ }^{6}$ \& MEGARA Team \\ ${ }^{1}$ Instituto Nacional de Astrofísica, Óptica y Electrónica; Luis Enrique Erro \# 1, Tonantzintla, Puebla, \\ 72840 , México \\ ${ }^{2}$ FRACTAL SLNE, C/Tulipán 2, Portal 13 1A, 28231, Las Rozas de Madrid, Spain \\ ${ }^{3}$ GUAIX group, Dept. Astrofísica Universidad Compluetense de Madrid, Av. Complutense s/n, \\ 28040, Madrid, Spain \\ ${ }^{4}$ Centro de Investigaciones en Óptica, Loma del Bosque 115, León, Guanajuato, México \\ ${ }^{5}$ Universidad Politécnica de Madrid, Madrid, Spain \\ ${ }^{6}$ Instituto de Astrofísica de Andalucía, Glorieta de Astronomía s/n, 18008, Granada, Spain
}

\begin{abstract}
MEGARA is the next optical Integral-Field Unit (IFU) and Multi-Object Spectrograph (MOS) for Gran Telescopio Canarias. The instrument offers two IFUs plus a Multi-Object Spectroscopy (MOS) mode: a large compact bundle covering $12.5 \operatorname{arcsec} \times 11.3$ arcsec on sky with $100 \mu \mathrm{m}$ fiber-core; a small compact bundle, of $8.5 \operatorname{arcsec} \times 6.7 \operatorname{arcsec}$ with $70 \mu \mathrm{m}$ fiber-core and a fiber MOS positioner that allows to place up to 100 mini-bundles, 7 fibers each, with 100 $\mu \mathrm{m}$ fiber-core, within a 3.5 arcmin $\times 3.5$ arcmin field of view, around the two IFUs. The fibers, organized in bundles, end in the pseudo-slit plate, which will be placed at the entrance focal plane of the MEGARA spectrograph. The large IFU and MOS modes will provide intermediate to high spectral resolutions, $\mathrm{R}=6800-17000$. The small IFU mode will provide $\mathrm{R}=8000-20000$. All these resolutions are possible thanks to a spectrograph design based in the used of volume phase holographic gratings in combination with prisms to keep fixed the collimator and camera angle. The MEGARA optics is composed by a total of 53 large optical elements per spectrograph: the field lens, the collimator and the camera lenses plus the complete set of pupil elements including holograms, windows and prisms. INAOE, a partner of the GTC and a partner of MEGARA consortium, is responsible of the optics manufacturing and tests. INAOE will carry out this project working in an alliance with CIO. This paper summarizes the status of MEGARA spectrograph optics at the Preliminary Design Review, held on March 2012.
\end{abstract}

Keywords: astronomical spectrographs, integral field units, multi-object fiber spectroscopy

\section{INTRODUCTION}

MEGARA stands for "Multi-Espectrógrafo en GTC de Alta Resolución para Astronomía" and meets the requirements of the Announcement of Opportunity for the new instrumentation for the Gran Telescopio Canarias, issued by GRANTECAN in 2009. It is an instrument project lead by the Universidad Complutense Madrid (UCM) in partnership with the Instituto Nacional de Astrofísica, Óptica y Electrónica (INAOE), the Instituto de Astrofísica de Andalucía (IAA) and the Universidad Politécnica de Madrid (UPM). INAOE will develop this project in collaboration with the Centro de Investigaciones en Óptica (CIO). The scientific motivations of the MEGARA science team members can be grouped in two categories: (1) the study of Galactic and extragalactic nebulae and (2) the study of (or close to) pointsources with intermediate-to-high surface density. An overview of the instrument and a more detailed description of the science drivers can be found in Gil de Paz et al. 2012 [1].

The Large Compact Bundle (LCB) covers $12.5 \operatorname{arcsec}$ x 11.3 arcsec on sky with $100 \mu \mathrm{m}$ fiber-core and 0.62 arcsec spaxel size. The Small Compact Bundle (SCB) covers $8.5 \operatorname{arcsec}$ x $6.7 \operatorname{arcsec}$ with $70 \mu \mathrm{m}$ fiber-core and 0.42 spaxel size. The fiber MOS positioner is able to place up to 100 mini-bundles, 7 fibers each, with $100 \mu \mathrm{m}$ fiber-core, within a

Ground-based and Airborne Instrumentation for Astronomy IV, edited by lan S. McLean, Suzanne K. Ramsay, Hideki Takami, Proc. of SPIE Vol. 8446, 84465B · @ 2012 SPIE · CCC code: 0277-786X/12/\$18 · doi: 10.1117/12.926694 
$3.5 \operatorname{arcmin} \times 3.5$ arcmin field of view (FoV) around the two fiber bundles. All the fiber bundles are placed on the foldedCassegrain focal station and are coupled to identical microlenses that convert the GTC f/17 into the $f / 3$ beam needed for an optimum use of the fibers by minimizing the focal ratio degradation (FRD). All the bundles go to a spectrograph that ideally will be placed on the Nasmyth platform. The spectrograph is composed by a pseudo-slit, where the fibers are placed simulating a long slit; a collimator, a $160 \mathrm{~mm}$ pupil where the volume phase holographic (VPH) gratings are placed, and the camera with the detector. The optics manufacturing will be carried out at INAOE \& CIO. Here we present the high level requirements, the design rationale and the optical preliminary design. This design was presented at the Preliminary Design Review held on March 2012. The previous spectrograph design developed during the Conceptual Design Phase is described by Carrasco et al. 2011 [2].

\section{OPTICAL DESIGN}

\subsection{Design rationale}

The main characteristics of the spectrograph and the high level requirements are summarized in Table 1.

Table 1. A subset of MEGARA main characteristics and spectrograph high level requirements (RQ).

\begin{tabular}{|c|c|}
\hline Filed of View & $\begin{array}{l}12.5 \times 11.3 \operatorname{arcsec}^{2} \text { (LCB) } \\
3.5 \times 3.5 \operatorname{arcmin}^{2} \text { (MOS-simultaneously with LCB) }\end{array}$ \\
\hline \# of spectrographs & Two (one approved) \\
\hline \# of spaxels / multiplexing & 5600 \\
\hline GTC station & Folded-Cass (spectrographs@Nasmyth) \\
\hline RQ-1. Wavelength range & $3700-9800 \AA$ \\
\hline RQ-2 Spaxel size & $0.62 \operatorname{arcsec}(\mathrm{LCB} \& \mathrm{MOS}) ; 0.42 \operatorname{arcsec}(\mathrm{SCB})$ \\
\hline RQ-3 Spectral resolution & $\mathrm{R}=5600-17000$ \\
\hline RQ-4 Detector format & $4096 \times 4096$ \\
\hline RQ-5 Pixel size & $15 \mu \mathrm{m} \times 15 \mu \mathrm{m}$ \\
\hline RQ-6 Fiber core diameter & $100 \mu \mathrm{m}(\mathrm{LCB} \& \mathrm{MOS}$ modes$)$ \\
\hline RQ-7 Image quality & $\mathrm{EED}_{80}$ in the resolution element of 4pix; goal: FWHM in 3.2 pix \\
\hline RQ-8 Entrance f-number of the spectrograph & $\mathrm{f} / 3$ \\
\hline RQ-9 Space between two adjacent fibres & 2 pixels \\
\hline
\end{tabular}

With these requirements we developed the following design rationale:

- Wavelength range: the spectrograph will have a single arm i.e. the whole range will be observed with the same optics.

- Spaxel size: the plate scale sets the fcoll/fcam ratio. As 0.62 arcsec has selected on the sky and the spectral element is required to be sampled with 4 pixels, we have considered the $0.62 \operatorname{arcsec}$ projected in 3.33 pixels giving a final plate scale of $0.188 \mathrm{arcsec} / \mathrm{pixel}$ on the detector. The total of 4 pixels is considered to be available for Error Budget (EB) margin.

- Scale Reduction factor: we will project the fiber size core $(100 \mu \mathrm{m})$ in 3.33 pixels by design. As the pixel size is $15 \mu \mathrm{m}$ wide, 3.3 pixels are $50 \mu \mathrm{m}$, what implies a scale reduction between the telescope focal plane and the detector of a factor of 2 . This factor will fix the ration between the f-numbers of collimator and camera.

- Collimator f-number: the spectrograph entrance f-number is chosen to minimize the FDR of the fiber link, thus $f / 3$ is used at the entrance and exit of the fiber becoming the collimator $f / \#$.

- Camera f-number: since the reduction factor between the telescoper focal plane is 2 and the collimator $\mathrm{f} / 3$ then the camera is $\mathrm{f} / 1.5$. 
- Pupil size: the current design has a $160 \mathrm{~mm}$ pupil diameter determined by the FoV. As we move to wider FoV the total focal length of the collimator and camera has to be increased in order to maintain the image quality. With the current experience filling 2000 pixels in the spatial direction will require a minimum pupil size of 80 $\mathrm{mm}$ and filling 4000 pixels in spatial direction will require a minimum of $160 \mathrm{~mm}$ pupil in order to manage field aberrations. This pupil size has been taken as reference for this design and this will limit the maximum FoV at the entrance of the spectrograph i.e. at the pseudo-slit.

- Spectral resolution and geometry: The grating equation:

$$
\frac{d \beta}{d \lambda}=\frac{m n}{\operatorname{COS} \beta}
$$

for the angular dispersion, sets the geometry of the light angle of incidence (AoI) on the grating, $\lambda$ is the wavelength; $\beta$ is the AoI on the grating, $\mathrm{m}$ the number lines $/ \mathrm{mm}$ and $\mathrm{n}$ the diffraction order. The best VPH performance regarding efficiency is normally obtained at first order $n=1$. This means that setting a HR mode will require a high AoI on the grating, while LR mode requires a low AoI on the grating. An example is shown in Figure 1.

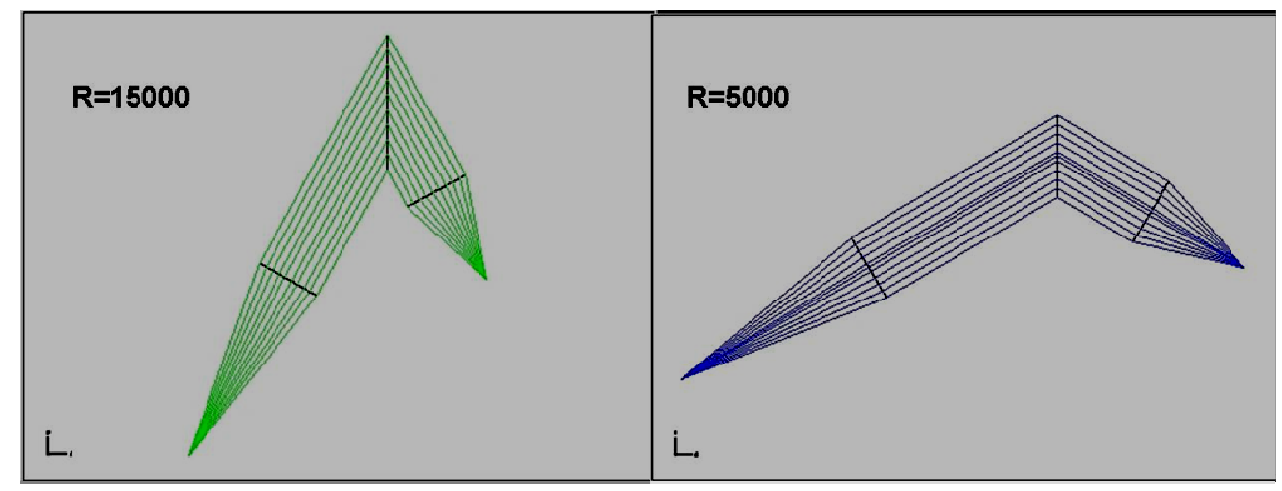

Figure 1: Example to illustrate the different angle on the grating needed for two extreme spectral resolution.

Different solutions can be offered for fitting a wide range of spectral resolutions:

(a) To consider two spectrographs geometries i.e. everything is the same except the envelope and the fixed geometry. This means to have two different spectrographs, one specific for HR and the other one for LR. This is optimum in terms of optical design.

(b) A single spectrograph with an articulated camera-grating that changes the geometry. In this case, the instrument would require a higher envelope and motorization of the grating and the camera-detector stage, what is not desirable. GRANTECAN requested a particular study on this issue and after a final review milestone, GRANTECAN agreed with MEGARA Team that the best solution was a fixed angle nonarticulated camera.

(c) A single spectrograph with a fixed geometry between the HR and the LR optimum angles. The final geometry on the grating is obtained by sandwiching the gratings between two prisms, what will change the angle of the beam to the required one.

All these previous options share the same optical design regarding the collimator and camera design. However, some differences are found regarding the gratings, and we have analyzed them in order to arrive to the Baseline configuration leading us to conclude that option (c) is the best one. Additonally, we included a novel design 
for the highest resolution mode based on segmented pupil gratings, described in detailed by Sáncchez-Blanco et al. $2011[3]$.

A detailed trade-off analysis showing the possible alternatives to widen the spectral resolution range and/or the wavelength coverage at the longest wavelengths was given as requested by GRANTECAN. The delivered study was reviewed by two independent persons selected by GRANTECAN. After their comments and recommendations and the meeting review, it was decided to close the MEGARA Optics concept with a fixed angle between collimator and camera. Therefore the possibility of using an articulated camera has been definitively discarded.

- Spectral elements: the VPHs are proposed as the spectral dispersers of the instrument. Ruled gratings are not available with the number of lines $/ \mathrm{mm}$ required while surface holographic gratings are not so efficient. Moreover transmission gratings are preferred over reflective ones because of the smaller size of the camera optics, as because in this case camera elements can be located very close to the dispersive element.

In the VPHs, the index pattern (hologram) is photo-recorded in dichromate gelatin. The hologram parameters, lines $/ \mathrm{mm}$ and AoI on the grating, give the wavelength that meets the Bragg condition. These values provide the required resolutions for the proposed gratings. The hologram thickness can be tuned to avoid a high dependence in the efficiency when out of Littrow angle.

The angle of incidence within the gelatin is given by the standard grating equation. Where $m=1$ is the order, $\lambda$ is the Bragg wavelength, $d$ is the grating spacing, and $n=1.27$ is the gelatin index.

$$
2 d n \operatorname{SIN} \beta_{m}=m \lambda
$$

The overall geometry implies that the required working angles will be around $26^{\circ}$ in the LR, around $48^{\circ}$ in the MR and around $70^{\circ}$ in the HR.

In order to change the AoI from a fixed geometry to the different angles required, prisms are used. The grating will be sandwiched inside. The prisms material will depend on the grating AoI. When the light goes from the gelatin to the material substrate or vice versa, we apply the Snell law.

$$
\mathrm{n}_{\text {gelatin }} \mathrm{x} \sin \beta=\mathrm{n}_{\text {prism }} \mathrm{x} \sin \alpha
$$

- Spectral resolution and fiber size: there is another way of changing the spectral resolution and this is by using different fiber sizes for different resolutions. For example if we use $100 \mu \mathrm{m}$ core fibers for $\mathrm{R}=10,000$ (projecting 1 fiber on 4 pixels) and $50 \mu \mathrm{m}$ for $\mathrm{R}=20,000$ (projecting 1 fiber on 2 pixels) we could have both resolutions with the same diffraction element. However, we initially discarded this solution for the MEGARA Baseline because it is desirable to use the same type of fibers to have the same spaxel on sky with all the resolutions and also the plate scale on sky covered by a $50 \mu \mathrm{m}$ fiber is very small $(0.3$ arcsec $)$ implying a subseeing sampling. The trade-off alternative study proposed as alternative the use of additional bundles with fiber core of $70 \mu \mathrm{m}$ to reach a higher spectral resolution in these additional bundles. This option was considered as add-on and allowed to introduce the small compact bundle of $\sim 500$ fibers $70 \mu \mathrm{m}$ fiber core. The spectrograph design was be done for the LCB while the SCB will be used with the same gratings as the LCB and MOS, providing a higher spectral resolution.

\section{BASELINE OF THE SPECTROGRAPH OPTICS DESIGN}

After studying different configurations during the Feasibility Study we decided to choose a fully refractive system for the spectrograph. The spectrograph is composed by a pseudo-slit, where the fibers are placed simulating a long slit. A collimator is composed by five lenses: one singlet and two doublets. The $160 \mathrm{~mm}$ diameter pupil is the location for 
VPH-gratings. In the preliminary design we proposed to mount the order sorting filter when needed together with the grating in the opto-mechanical mount. Once the beam passes through the grating it goes to the camera, composed by two doublets and three singlets, and then to the detector. We are considering that the last lens is also the cryostat window. The MEGARA team has experience with this configuration that offers the advantage of increasing the throughput by saving two surfaces.

\subsection{Spectrograph subsystems}

\subsubsection{Pseudo slit}

Main characteristics: the slit length is $119 \mathrm{~mm}$, curved on a sphere surface of ROC $1075 \mathrm{~mm}$. The fibers mounted on the pseudo slit will form a regularly spaced linear array. This assembly in-line is acceptable since the scientific requirements of having 2 projected pixels on the detector between adjacent fibers to avoid cross-talking is equivalent to have a pitch of $178 \mu \mathrm{m}$, so that having $170 \mu \mathrm{m}$ pitch has been accepted. The final number of fibers in the pseudo-slit will be decided after the final pseudo slit design but we are estimating to have a number around 623 fibers for LCB, 644 fibers for MOS and 500 for SCB modes.

\subsubsection{Collimator}

Main characteristics: f/3, focal length $483.3 \mathrm{~mm}$. The layout is shown in Figure 2 and the optical parameters in Table 2. The only aspheric that we are using in the optical design is the COLL-S1 element. This is a high order aspheric, which characteristics are shown in Figure 3. The focussing mechanism shown in the first doublet of Figure 2 might be changed to the slit location.

\subsubsection{Pupil}

The pupil size is $160 \mathrm{~mm}$, on average, and the gratings will be located in this position.

\subsubsection{Geometry Collimator-Camera}

Fixed. $68^{\circ}$ between the collimator and the camera.

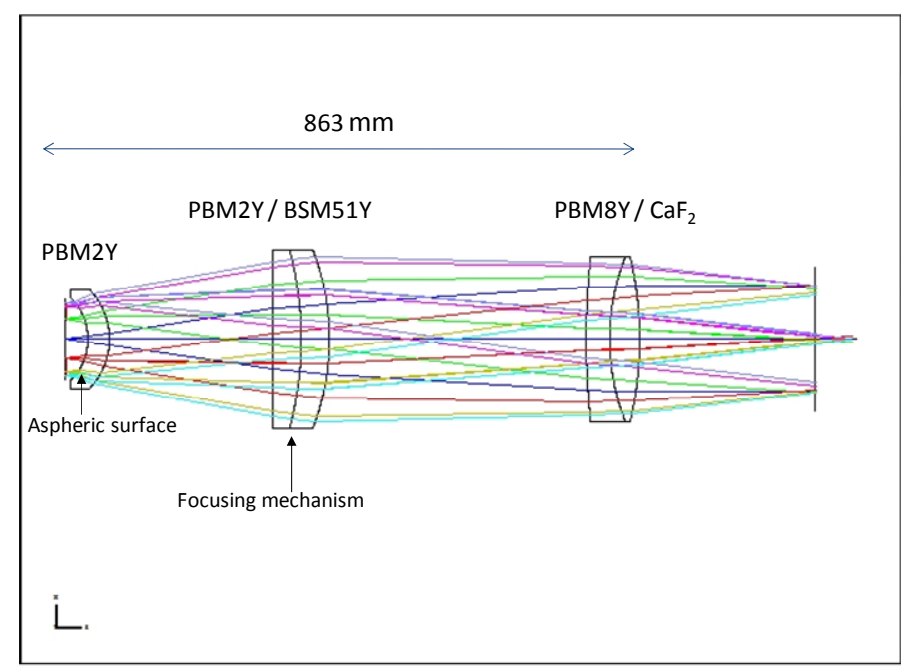

Figure 2: Collimator layout. It is composed by an aspheric singlet and two doublets. 
Table 2: Collimator lenses parameters. COLL D2-COLL D3 and COLL D4-COLL D5 are doublets

\begin{tabular}{|l|c|c|c|c|c|}
\hline \multicolumn{7}{|c|}{ Collimator Optical Elements } \\
\hline Element & Material & R1 (mm) & R2 (mm) & Central Thickness (mm) & $\begin{array}{c}\text { Blank } \varnothing \\
\text { Estimation (mm) }\end{array}$ \\
\hline COLL S-1 & PBM2Y & $-91.37(*)$ & -108.8 & 32.5 & 160.0 \\
\hline COLL D-1 & PBM2Y & flat & -529.7 & 42.0 & 272.0 \\
\hline COLL D-2 & BSM51Y & -529.7 & -389.1 & 42.0 & 272.0 \\
\hline COLL D-3 & PBM8Y & +1197.8 & +346.3 & 35.0 & 252.0 \\
\hline COLL D-4 & CaF2 & +346.3 & -563.6 & 45.0 & 250.0 \\
\hline
\end{tabular}

(*) high order aspheric

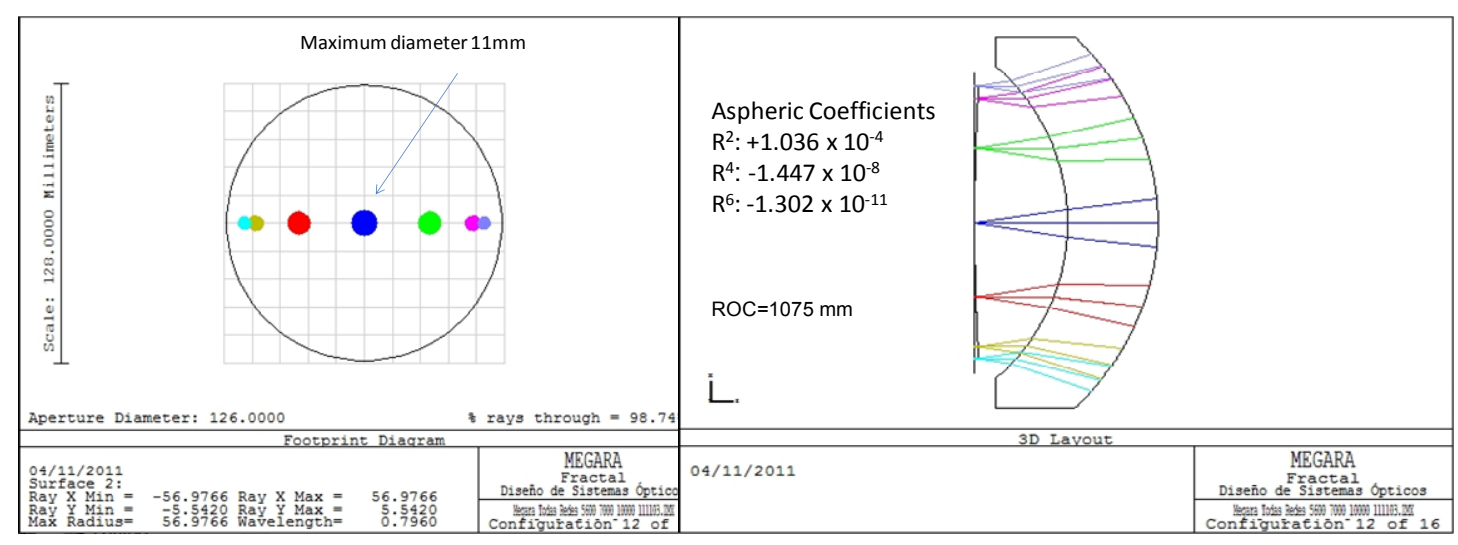

Figure 3: Aspheric COLL S-1 element. The optical parameters are shown on the right and footprint diagram is on the left.

\subsubsection{Camera}

The main characteristics are: $\mathrm{f} / 1.5$, focal length $245.9 \mathrm{~mm}$, image field is $61.4 \mathrm{~mm} \times 61.4 \mathrm{~mm}$ covering $4 \mathrm{~K} \times 4 \mathrm{~K}$ pixels. The camera is composed by two doublets and 3 singlets as seen in Figure 4. The characteristics of the camera lenses are summarized in Table 3.

The total expected transmission of the optical system, excluding the pupil elements is shown in Figure 5. This transmission expectative is very good and very high for such a complex spectrograph. The optimization of the pupil elements will be done in a later phase and, as far as they will be used in a fixed angle, could be optimized for each wavelength range. INAOE and CIO are studying this coating issue. These data have not been updated with the first collimator lens material that is 1 per cent better in the blue. Moreover, the data have been calculated assuming 1.5 per cent Fresnel losses per glass/air interface and this implies that this is the worse case. INAOE and CIO are working in providing coatings better than 1.3 per cent. 


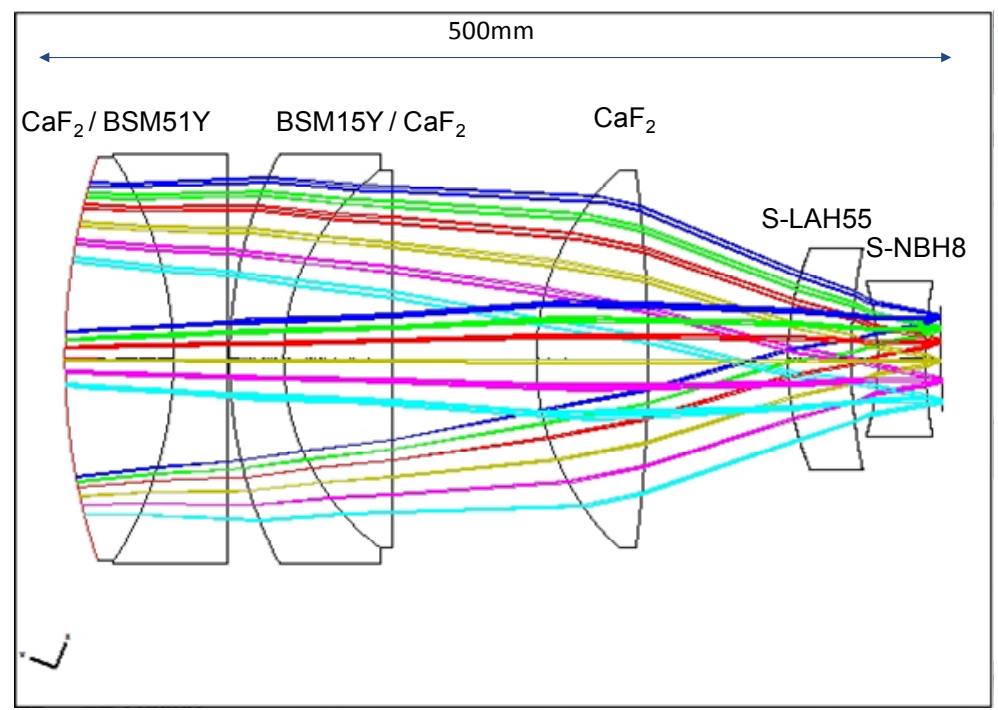

Figure 4: Camera layout. The last lens is the cryostat window.

Table 3: Camera lenses parameters. CAM D1 - CAM D2 and CAM D3 - CAM D4 are doublets element..

\begin{tabular}{|c|c|c|c|c|c|}
\hline \multicolumn{7}{|c|}{ Camera optical elements } \\
\hline Element & Material & $\begin{array}{c}\text { R1 } \\
(\mathrm{mm})\end{array}$ & $\begin{array}{c}\text { R2 } \\
(\mathrm{mm})\end{array}$ & $\begin{array}{c}\text { Central } \\
\text { Thickness } \\
(\mathrm{mm})\end{array}$ & $\begin{array}{c}\text { Blank } \varnothing \\
\text { Estimation } \\
(\mathrm{mm})\end{array}$ \\
\hline CAM D-1 & $\mathrm{CaF}_{2}$ & 391 & -227 & 60.0 & 210.0 \\
\hline CAM D-2 & BSM51Y $_{\text {CAM D-3 }}$ & -227 & Flat & 30.0 & 220.0 \\
\hline BAL15Y & 273 & 140 & 30.0 & 220.0 \\
\hline CAM S-5 & CaF $_{2}$ & 140 & Flat & 60.0 & 215.0 \\
\hline CAM S-6 & CaF $_{2}$ & 155 & -887 & 62.0 & 215.0 \\
\hline CAM S-7 & S-LAH55 & 175 & 329 & 35.1 & 120.0 \\
\hline
\end{tabular}

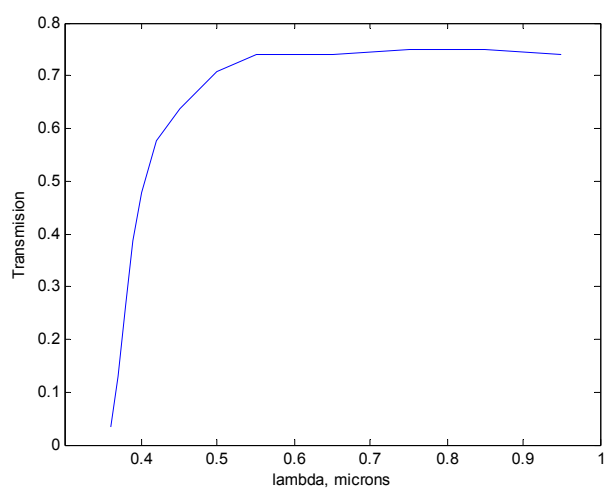

\begin{tabular}{|c|c|}
\hline Lambda $(\AA)$ & Transmission \\
\hline 3600 & 0.0343 \\
3700 & 0.1295 \\
3800 & 0.2636 \\
3900 & 0.3861 \\
4000 & 0.4781 \\
4200 & 0.5772 \\
4500 & 0.6380 \\
5000 & 0.7067 \\
5500 & 0.7402 \\
6500 & 0.7402 \\
7500 & 0.7488 \\
8500 & 0.7504 \\
9500 & 0.7410 \\
\hline
\end{tabular}

Figure 5: Plot and values of the predicted transmission of the collimator and camera optics. 


\subsubsection{Pupil elements}

Different types of pupil elements, all of them based on VPH-type gratings, can be accommodated in the pupil position to provide the different spectral modes, with resolution power ranging between 5500 and 17000 . Low resolution units will be built with simple gratings sandwich between two flat windows; mid units are provided with the gratings sandwiched between two symmetric prisms that allow the beam to incident on the VPH in the optimum angle. This solution is feasible and well tested and it was the solution used for the instruments DOLORES for the TNG and Elmer for the GTC. For the high resolution we propose to apply a novel design of sliced pupil grating detailed described by Sánchez-Blanco et al. 2012, [3]. Figure 6 shows the different MEGARA modes (low, medium and high) with the AoI fixed to $\alpha=34^{\circ}$ and different pupil elements.

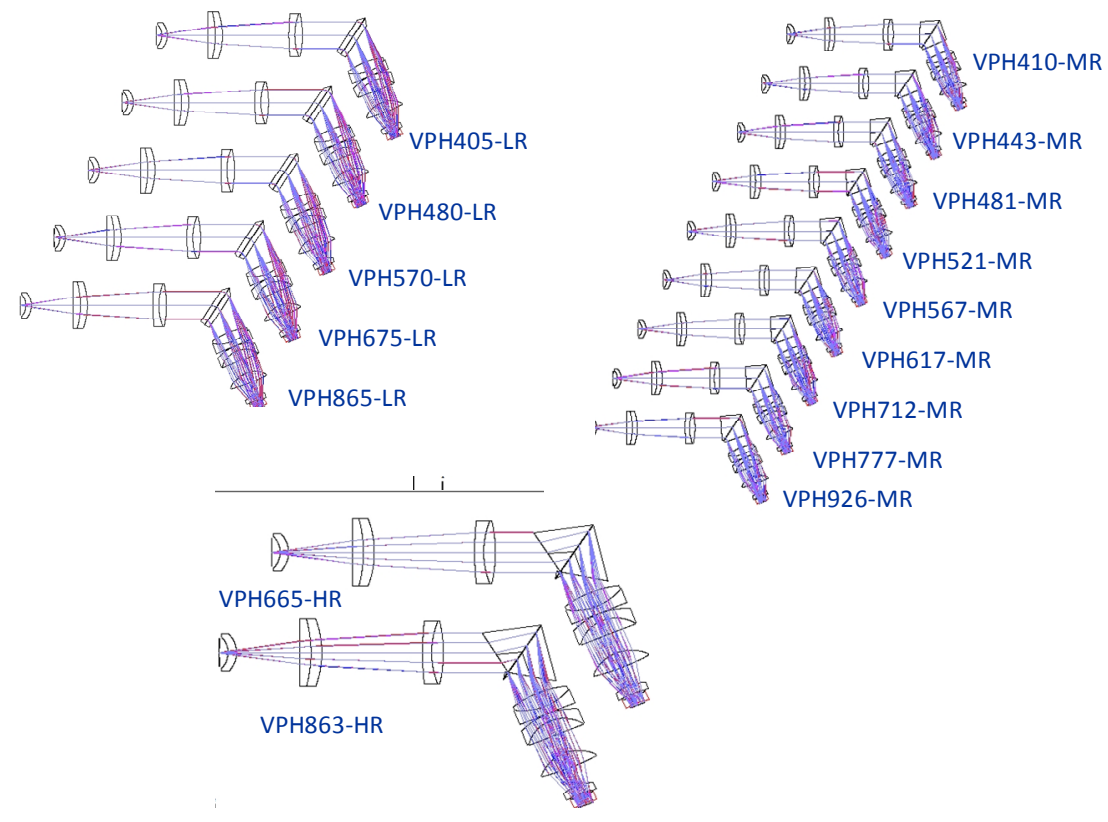

Figure 6: MEGARA Baseline spectrograph: this configuration keeps fixed the angle between the collimator and the camera. The lowresolution mode $(\mathrm{R}=5,500)$ uses at pupil a VPH grating sandwiched between two flat windows. The different angles on the hologram needed for the different spectral resolutions are obtained by sandwiching the holograms between two prisms $($ at $\mathrm{R}=10,000)$ and by segmenting the pupil in two slices with two prisms placed at each side of the hologram (in the case of $\mathrm{R}=17,000$ ).

The spectral resolutions of MEGARA Baseline are $\mathrm{R}=5500$ in the whole range, $\mathrm{R}=10000$ in the complete blue range, only limited by budget to provide additional optical-red coverage and $\mathrm{R}=17000$ in selected ranges defined by the science team (centered at $\mathrm{H} \alpha 6563 \AA$ and the $\mathrm{CaT}$ lines at $8500-8700 \AA$ ). These values are referred to $\mathrm{EED}_{80}$. A maximum of 20 per cent of different resolution is allowed due to the angle of $7.2^{\circ}$ to see the whole camera. This margin depends on resolution and is well within the scientific requirements. The complete list of MEGARA gratings scientific requirement is given in Gil de Paz et al. 2012 [1]. Table 4 presents the selected grating parameters and size. The spectral resolution 
values in this table are all referred to $\mathrm{EED}_{80}$, which was the only parameter used for image quality requirement and performance evaluation during the Preliminary Design Phase.

Table 4: MEGARA Baseline gratings: VPH specifications.

\begin{tabular}{|c|c|c|c|c|c|c|}
\hline VPH Name & $\mathbf{R}_{\text {EED80 }}$ & $\begin{array}{c}\boldsymbol{\lambda} \text { Bragg } \\
\boldsymbol{\AA}\end{array}$ & $\begin{array}{c}\boldsymbol{\theta} \text { Bragg } \\
\text { degrees }\end{array}$ & $\begin{array}{c}\text { Record } \\
\text { lines/mm }\end{array}$ & $\begin{array}{c}\text { Prism Apex } \\
\text { degrees }\end{array}$ & $\begin{array}{c}\text { Optical Aperture } \\
\mathbf{m m} \mathbf{\text { mm }}\end{array}$ \\
\hline VPH405-LR & 5,473 & 4,051 & 26.12 & 2,761 & Flat & $172 \times 200$ \\
\hline VPH480-LR & 5,504 & 4,800 & 26.12 & 2,330 & Flat & $172 \times 200$ \\
\hline VPH570-LR & 5,523 & 5,700 & 26.12 & 1,962 & Flat & $172 \times 200$ \\
\hline VPH675-LR & 5,533 & 6,749 & 26.12 & 1,657 & Flat & $172 \times 200$ \\
\hline VPH865-LR & 5,559 & 8,650 & 26.12 & 1,293 & Flat & $172 \times 200$ \\
\hline VPH410-MR & 10,000 & 4,104 & 47,65 & 4,574 & 35.769 & $172 \times 205$ \\
\hline VPH443-MR & 10,000 & 4,431 & 47,99 & 4,259 & 36.863 & $172 \times 205$ \\
\hline VPH481-MR & 10,000 & 4,814 & 48,22 & 3,935 & 37.745 & $172 \times 205$ \\
\hline VPH521-MR & 10,000 & 5,213 & 48,39 & 3,643 & 38.420 & $172 \times 205$ \\
\hline VPH567-MR & 10,000 & 5,667 & 48,54 & 3,359 & 39.026 & $172 \times 205$ \\
\hline VPH617-MR & 10,000 & 6,170 & 48,64 & 3,090 & 39.494 & $172 \times 205$ \\
\hline VPH712-MR & 10,000 & 7,115 & 48,82 & 2,685 & 40.231 & $172 \times 205$ \\
\hline VPH777-MR & 10,000 & 7,767 & 48,76 & 2,459 & 40.335 & $172 \times 205$ \\
\hline VPH926-MR & 10,000 & 9,262 & 48,65 & 2,066 & 40.486 & $172 \times 205$ \\
\hline VPH665-HR & 17,000 & 6,646 & 70,41 & 3,601 & 68.201 & $172 \times 230$ \\
\hline VPH863-HR & 17,000 & 8,634 & 69,87 & 2,762 & 68.673 & $172 \times 230$ \\
\hline
\end{tabular}

The final specifications of the VPH shall be derived once the final optical detailed design is closed. The requirement of having both HR ( $R \sim 20000$, which is 17000 in the current configuration) and LR ( $R \sim 5500$ ) spectral resolutions implies very different values for the optimal angle of incidence on the VPHs. These angles need to vary between $26^{\circ}$ and $70^{\circ}$ for resolutions between 5500 and 17000 .

In order to cover the entire optical range, different VPHs are needed. MEGARA Baseline has a total of 5 low-resolution VPHs to cover the entire optical range from $3700 \AA$ to $9500 \AA$ at $R=5500,9$ mid-resolution VPHs at $\mathrm{R}=10000$ to cover the entire range except the ranges already covered at high resolution, and 2 high-resolution gratings around the $\mathrm{H}$ alpha and Calcium triplet $n$ ranges at $\mathrm{R}=17000$. In the current configuration we have defined the wheel with 11 positions so that we would mount 5 LR gratings, 4 MR gratings, the most widely-used ones, and 2 HR gratings. In an alternative configuration all $9 \mathrm{MR}$ and $2 \mathrm{HR}$ gratings could be mounted simultaneously yielding full optical coverage to MEGARA at $\mathrm{R}\left(\mathrm{EED}_{80}\right)=10000-17000$. Having the possibility of these two configurations is a requirement for the wheel design.

\section{IMAGE QUALITY}

\subsection{Image quality requirement}

The image quality requirement is to have the resolution element in four pixels i.e. $60 \mu \mathrm{m}$. The value that contains the $80 \%$ of the encircled energy $\left(\mathrm{EE}_{80}\right)$ coming from a fiber whose projection is $50 \mu \mathrm{m}$ is a diameter of $34.32 \mu \mathrm{m}$, as illustrated in Figure 7. Thus,

$$
\mathrm{EE}_{80}=60^{2}-34.32^{2}=49.2^{2}
$$


For this reason the total value of $\mathrm{EE}_{80}$ through the system has to be lower than $49.2 \mu \mathrm{m}$ a in diameter or $\mathbf{2 4 . 6} \boldsymbol{\mu m}$ in radius. In other words, this $24.6 \mu \mathrm{m}$ in radius is the available budget to degrade the image quality. From the previous calculations the value of the requirement when analyzing the Image performance will have a value of $\mathrm{EER}_{80} \leq 24.6 \mu \mathrm{m}$.

We analyzed the image quality performance obtained with the different configurations of the instrument i.e. for different spectral resolutions. For each grating we obtained the projection of the spectra and the spot diagram at different fiber positions on the pseudo-slit, covering the whole pseudo-slit length and therefore the whole range of AoI on the grating. Additionally, a total of six wavelengths were analyzed in each configuration providing a graph where the values of $E_{8 E}$ is plotted as a function of the position. An example of the plots obtained for each configuration is shown in figures 8, 9 and 10 for a VPH based on a grating with 1657 lines $/ \mathrm{mm} ; \mathrm{R}=5533$ and $\lambda \mathrm{c}=6749 \AA . \mathrm{R}=5600$. All the configurations are within requirements and with a good margin for error budget.

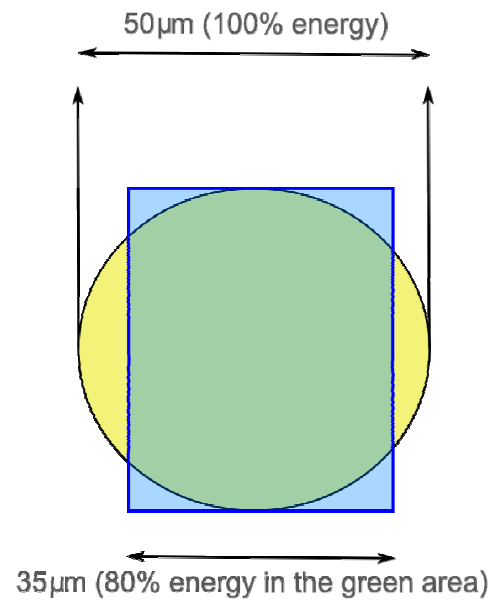

Figure 7. The value of the diameter of a circle that contains the $80 \%$ of the energy from a $100 \mu \mathrm{m}$ fiber, whose circular projection on the detector is a circle of a diameter of $50 \mu \mathrm{m}$, is $35 \mu \mathrm{m}$.

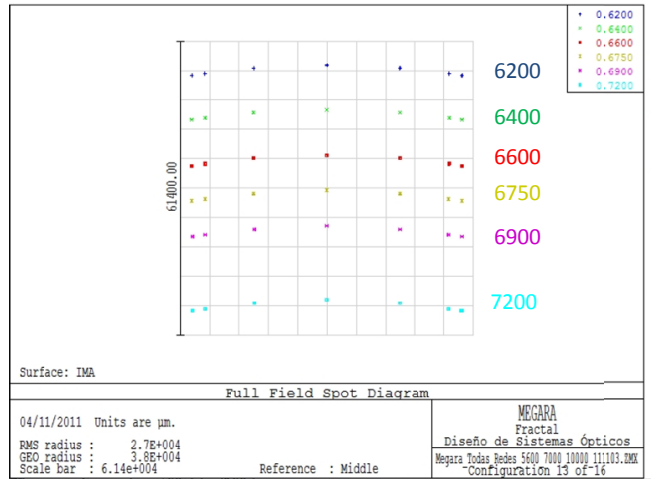

Figure 8: Projection on the detector of the spectra from fibers at different positions of the pseudo-slit in the configuration of the grating VPH675-LR. Different colors correspond to different wavelengths. 


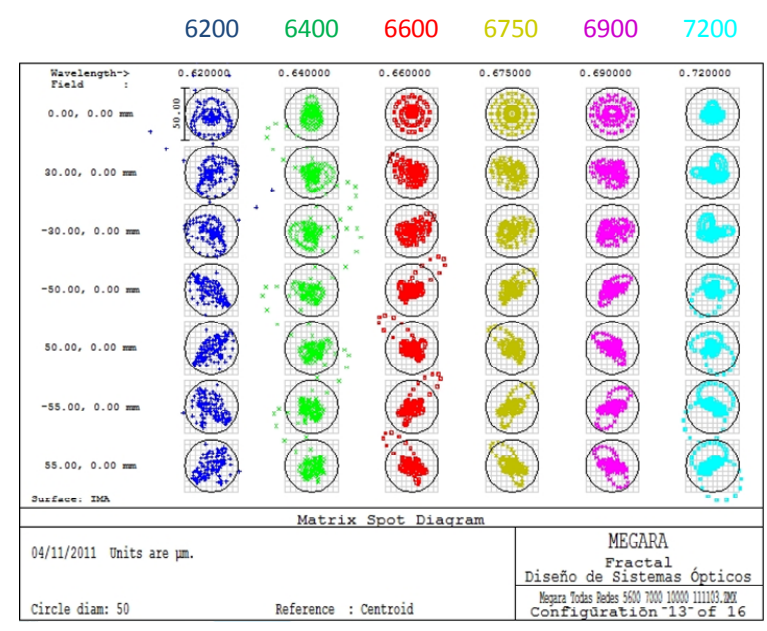

Figure 9: Spot diagram of the spectra from fibers at different positions of the pseudo-slit in the configuration VPH675-LR. Different colors correspond to different wavelengths.
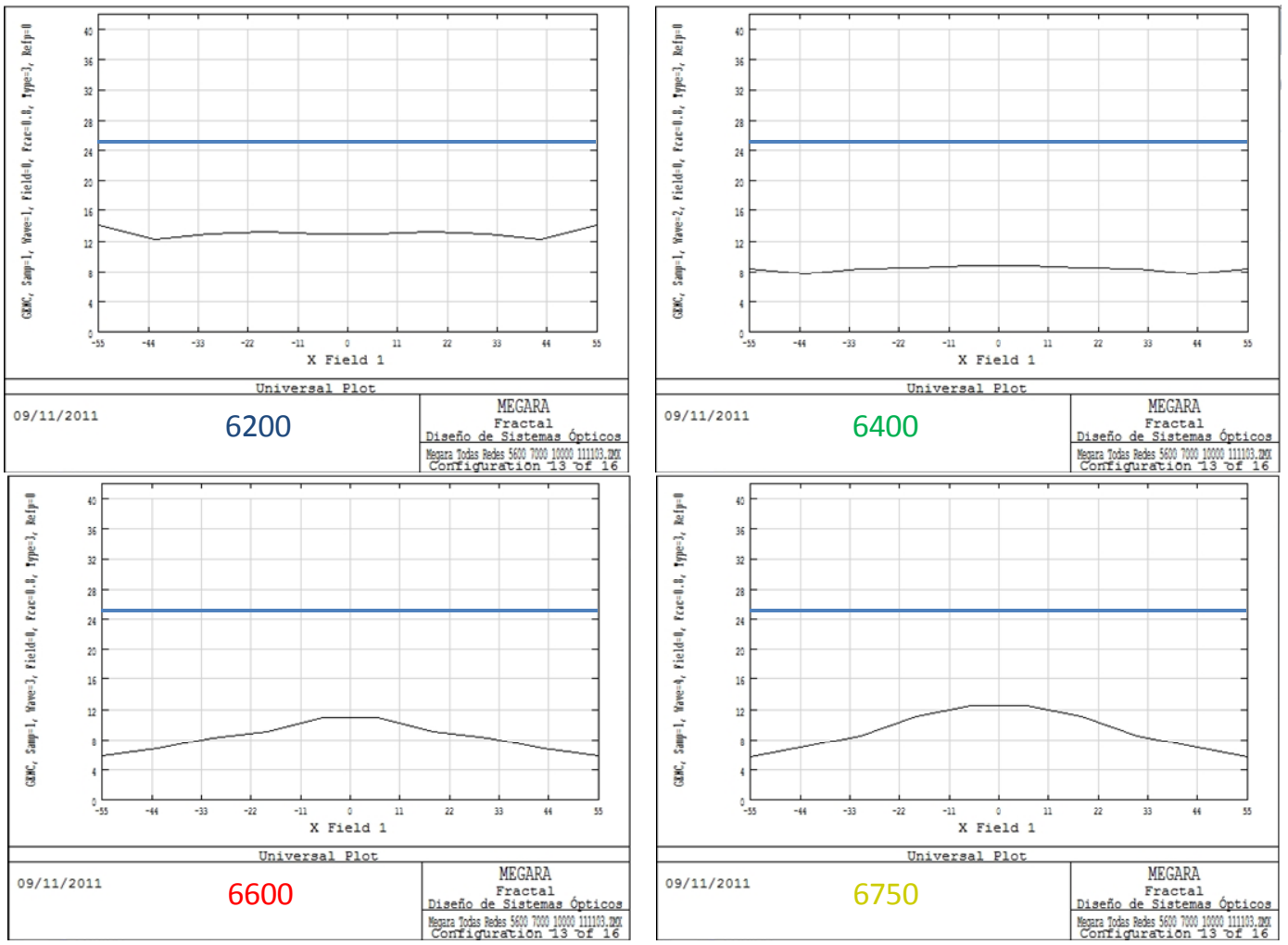

Figure 10: continues

Proc. of SPIE Vol. 8446 84465B-11 

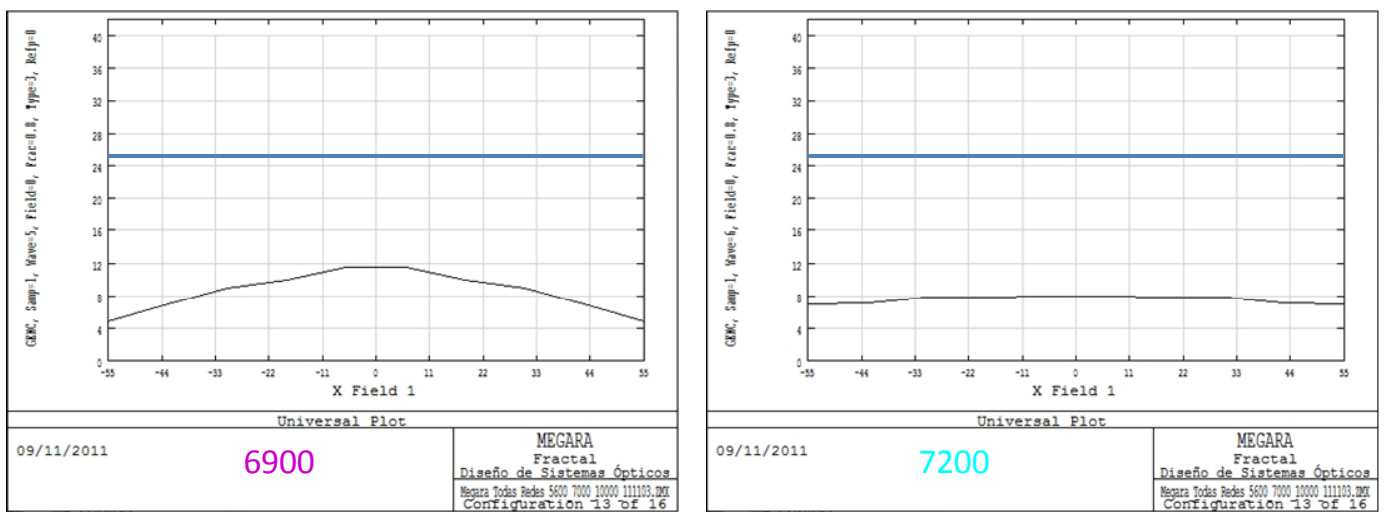

Figure 10: $\mathrm{EER}_{80}$ as a function of the position of the FoV (on the pseudo-slit) grating VPH675-LR. Different boxes have been evaluated in different wavelengths. The blue line indicates the image quality requirement. As seen in the figure, all fields and wavelengths are well within requirements.

\subsection{Image Quality Error Budget}

MEGARA performance has to be guaranteed after fabrication and assembly considering all the possible error sources. We analyze the error budget regarding image quality in one of the representative modes. The EB presented here was done in a previous issue of the design (October 2011). The new issue has only minor changes, some radii and thicknesses have been slightly changed. Also the material of COLL S-1 was changed due to unavailability of the blank material at the desired thickness. We will re-do the EB once more after all the final changes and suggestions implemented on the design presented at the PDR.

The errors associated to different tolerances were computed analytically or with Monte Carlo (MC) models. In the case of MC models a normal probabilistic distribution between the lower and upper limits was used and the associated error was considered at the 90 per cent level of occurrences, thus the current analysis expects that $90 \%$ of the systems will be within the current expectations.

All the budgeted items, except the fiber size itself, shall amount a maximum degradation value of $\mathrm{EED}_{80}<49.2 \mu \mathrm{m}$. For practical optical analysis purposes the evaluation of the $\mathrm{EB}$ will not be made in terms of $\mathrm{EED}_{80}$, but in terms of RMS spot radius, and considering 1d Gaussian profile in the spectral direction. Thus, in order to compute the EB, we will translate the $\mathrm{EE}_{80}$ to the $\sigma$ of a Gaussian. The $80 \%$ of the energy under a 1d Gaussian is contained under $\pm 1.28 \sigma$. Thus $\mathrm{EED}_{80}=49.2 \mu \mathrm{m}$, will become $\mathrm{EER}_{80}=24.6 \mu \mathrm{m}$ or

$$
\sigma=\mathrm{EER}_{80} / 1.28=19.22 \mu \mathrm{m} ;
$$

where $19.22 \mu \mathrm{m}=\sigma$ associated to $80 \%$ of EER. Table 6 summarizes the EB contributors. The budget is built:

$$
\sigma_{\text {total }}^{2}=\sigma_{\text {design }}^{2}+\sigma_{\text {fabrication }}^{2}+\sigma_{\text {alignment }}^{2}+\sigma_{\text {thermal }}^{2}+\ldots
$$

The error budget has to include all the contributors to image quality, and the quadratic sum of all of them has to reconstruct the requirement. The expected result for the $\sigma$ is $15.10 \mu \mathrm{m}$, while the total value is $19.22 \mu \mathrm{m}$. 
Table 6: Estimated values for the different Image Quality EB contributors for $100 \mu \mathrm{m}$ fiber core

\begin{tabular}{|c|c|c|}
\hline ITEM & $\sigma(\mu \mathrm{m})$ & Comment \\
\hline Nominal performance & 11.20 & Nominal design in one representative mode \\
\hline $\begin{array}{l}\text { Collimator fabrication (lens thickness, } \\
\text { wedge, surface irregularity, curvature) }\end{array}$ & 2.10 & 100 Monte Carlo runs in normal distribution \\
\hline $\begin{array}{l}\text { Camera fabrication (lens thickness, } \\
\text { wedge, surface irregularity, curvature) }\end{array}$ & 2.98 & 100 Monte Carlo runs in normal distribution \\
\hline $\begin{array}{l}\text { Coll AIV (axial and lateral decentration, } \\
\text { tilts) }\end{array}$ & 1.84 & 100 Monte Carlo runs in normal distribution \\
\hline $\begin{array}{l}\text { Camera AIV (axial and lateral } \\
\text { decentration, tilts). }\end{array}$ & 4.20 & 100 Monte Carlo runs in normal distribution \\
\hline Uncompensated & 5.00 & 100 Monte Carlo runs in normal distribution \\
\hline Thermal & 2.10 & Worst case. Analytical model \\
\hline Glass homogeneity & 1.70 & Analytical model \\
\hline Pupil elements & 5.00 & Allocated Estimation \\
\hline Detector Flatness & 1.32 & Considering flatness $\pm 5 \mu \mathrm{m}$ \\
\hline Pseudo-slit curvature & 3.04 & \\
\hline TOTAL (rms squared) & 15.10 & \\
\hline
\end{tabular}

\section{CONCLUSIONS}

We developed a preliminary optical design that fulfills all the scientific requirements. The design is consistent of an $\mathrm{f} / 3$ fiber fed spectrograph, projected onto a $4 \mathrm{k} \mathrm{x} 4 \mathrm{k}$ detector and with a resolution element of 4 pixels for a $100 \mu \mathrm{m}$ fiber core, which is the case for the LCB and MOS modes. The design is based on a collimator-camera system with a fixed angle between the two elements, that has been chosen to allow the wide spectral resolution range required in the scientific requirements. The presented design covers a range between $\mathrm{R}=5600$ and 17000 that has been optimized according to: (a) GTC requirements, (b) the science team requirements and (c) the need of using a single detector what has been considered a design requirement.

Spectral resolution is obtained through VPH gratings. These VPH gratings will be sandwiched between two flat windows (in the $\mathrm{LR}$ mode at $\mathrm{R}=5600$ ) and two prisms (in the MR mode at $\mathrm{R}=10000$ ). In the case of the HR mode our baseline is 
to use a sandwich between two prisms each side, using our novel design of sliced pupil gratings. Other alternatives like two prisms sandwich covering the whole pupil are under study.

All the modes have room enough in the Image Quality Error Budget to assure that the high level scientific requirements will be fulfilled. Evaluation has been done for all VPHs defined by the scientific requirements and along the whole wavelength range and spatial distribution along the pseudo-slit. Here we presented the case of one configuration.

MEGARA has the Preliminary Design Review on March 2012. Regarding the spectrograph optics presented here the foreseen changes are minor.

\section{REFERENCES}

[1] A. Gil de Paz, E. Carrasco, J. Gallego, M. Sánchez, J.M. Vílchez, M.L. García-Vargas et al. "MEGARA: the future optical IFU and multi-object spectrograph for the $10.4 \mathrm{~m}$ GTC telescope" These Proceedings (2012).

[2] Carrasco, Esperanza; Sánchez-Blanco, Ernesto; García Vargas, María Luisa; Gil de Paz, Armando; Gallego, Jesús; Páez, Gonzalo; Zamorano, Jaime; Castro, Jorge. "Optical Design for MEGARA: a multi-object spectrograph for the GTC" 22nd Congress of the International Commission for Optics: Light for the Development of the World. Edited by Rodríguez-Vera, Ramón; Díaz-Uribe, Rufino. Proceedings of the SPIE, Volume 8011, pp. 80112D-80112D-10 (2011).

[3] Sánchez-Blanco, E.; García-Vargas, M.; Maldonado, M.; Gallego, J.; Gil de Paz, A.; Carrasco, E.; Pérez, A.; Martínez-Delgado, I.; Zamorano, J. "Sliced-pupil grating: a novel concept for increasing spectral resolution" 22nd Congress of the International Commission for Optics: Light for the Development of the World. Edited by Rodríguez-Vera, Ramón; Díaz-Uribe, Rufino. Proceedings of the SPIE, Volume 8011, pp. 80111I-80111I-10 (2011). 